\title{
The place of musculoskeletal ultrasonography in gout diagnosis
}

\author{
Daniela Fodor' ${ }^{\text {, Rodina Nestorova }}{ }^{2}$, Violeta Vlad ${ }^{3}$, Mihaela Micu 4 \\ ${ }_{1}^{1} 2^{\text {nd }}$ Internal Medicine Department, "Iuliu Hatieganu" University of Medicine and Pharmacy, Cluj-Napoca, Romania, \\ ${ }^{2}$ Centre of Rheumatology “St. Irina”, Sofia, Bulgaria, ${ }^{3}$ Sf. Maria Clinical Hospital, Bucharest, Romania, ${ }^{4}$ Rheumatol- \\ ogy Division, Department of Rehabilitation II, Rehabilitation Clinical Hospital, Cluj-Napoca, Romania
}

\begin{abstract}
Gout represents a common inflammatory arthritis conditions in which the monosodium urate (MSU) crystals are deposited in joints and other tissues producing attacks of acute arthritis/ chronic arthropathy with important destructive lesions in articular or juxta-articular spaces.

High resolution ultrasonography (US) is able to detect these deposits and their consequences: hyaline cartilage pathology, joint effusions, synovitis, bone erosions, tendonitis, bursitis, and other soft tissues involvement. Moreover, there are characteristic findings of this aggregates that can be detected by US, helping the clinician for positive and differential diagnosis. In this review we will present the main US findings in gout. The utility of the method in diagnosis and treatment response will be discussed. Also, comparison with other imaging techniques in terms of sensibility and specificity of the methods for this pathological situation will be made.
\end{abstract}

Keywords: gout, ultrasonography, double contour sign, monosodium urate aggregates, tophus

Gout is a term representing a heterogeneous group of metabolic disorder found exclusively in humans, characterized by increased serum urate concentration for a long period of time, resulting into tissular deposition of monosodium urate (MUS). Hippocrates described acute gout in the 4th century BC but Thomas Sydenham made the classic description of acute gout in the 17 th century.

Gout is a common inflammatory arthritis conditions, causing morbidity, disability, and poorer quality of life. The disease is frequently associated with other co-morbidities (type 2 diabetes mellitus, cardiovascular disease, chronic kidney disease, or obesity) [1]. It is more common in men that women and the prevalence increases with age. The gout is the consequence of deposition of

Received 22.09.2013 Accepted 18.10.2014

Med Ultrason

2014, Vol. 16, No 4, 336-344

Corresponding author: Daniela Fodor, MD, PhD

2nd Internal Medicine Department

"Iuliu Hatieganu" University of Medicine

and Pharmacy, Cluj-Napoca, Romania

2-4 Clinicilor str,

400006 Cluj Napoca, Romania

Phone: 004 0264591942/442

Email: dfodor@umfcluj.ro
MSU crystals in joints and other tissues as a result of persistent hyperuricaemia [2]. Traditionally, four phases are described in the disease evolution: asymptomatic hyperuricaemia, acute, intercritical, and chronic gout [3] but recently a new revised staging system for hyperuricaemia and gout was proposed (table I) [4].

Diagnosis of suspected gout is based on typical clinical and laboratory (ie, hyperuricaemia) findings, while definitive diagnosis requires identification of MSU crystals in aspirated synovial fluid or tophi [2]. The crystals of MSU are proved to be in and around the joints (car-

Table I. Proposed staging system for gout (from [4] adapted).

\begin{tabular}{lll}
\hline Stage & Symptomatology & Definition \\
\hline A & Asymptomatic & $\begin{array}{l}\text { Hyperuricaemia without MSU } \\
\text { crystal deposition } \\
\text { MSU crystal deposition but with- } \\
\text { out signs or symptoms of gout }\end{array}$ \\
C & Symptomatic & $\begin{array}{l}\text { MSU crystal deposition with prior } \\
\text { or current episodes of acute gout } \\
\text { flares }\end{array}$ \\
& & $\begin{array}{l}\text { Advanced gout (tophaceous gout } \\
\text { and chronic gouty arthropathy) } \\
\text { requiring specialized interventions }\end{array}$ \\
D & &
\end{tabular}


tilage, epiphyses, synovial membrane, tendons, or ligaments). These aggregated deposits produce local necrosis and, with the exception of avascular tissues, the inflammatory response can lead to a fibrous proliferation [5].

In the early stage of gout radiography is less useful for diagnosis due to the non-specific soft-tissue swelling overlying the inflamed joint; abnormal findings specific for radiology will generally appear after years of evolution [6]. $\mathrm{CT}$ and MRI have been used in few studies [7-10] especially to evaluate the tophi, but these methods cannot be used as routine examinations. Ultrasonography (US) instead, can be used for diagnosis and management of gout from the first attack. Crystalline material found in gouty joints reflects ultrasound waves more strongly than surrounding tissues (such as un-mineralized hyaline cartilage or synovial fluid) and can thus be readily distinguished [11].

The US findings in gout are specific or non-specific [6] and for some of these findings OMERACT defini- tions were recently established [12] (table II) (for some of the gout findings the previous OMERACT definitions should be used [13]).

The MSU crystals can precipitate theoretically in every anatomical structure but there are specific places where these deposits have to be searched (table III).

\section{Synovial fluid}

Effusions are less specific for gout. Intra-articular fluid can be strictly anechoic (simple joint effusion) at the first gouty attacks (early disease) but in time MSU aggregates with variable echogenicity will appear $[3,6,14-$ 17]. The hyperechoic spots have less than $1 \mathrm{~mm}$ and their presence within the synovial fluid realize the "starry sky" sign [18]. During the real time examination, at a delicate pressure with the transducer under the examined joint, the spots will float inside the joint cavity creating a "snow-

Table II. The ultrasonographic findings in gout and OMERACT definitions $[12,13]$.

\begin{tabular}{|c|c|}
\hline Specific findings & OMERACT definition $[12,13]$ \\
\hline Double contour sign & $\begin{array}{l}\text { "Abnormal hyperechoic band over the superficial margin of the articular hyaline cartilage, independent } \\
\text { of the angle of insonation and which may be either irregular or regular, continous or intermittent and can } \\
\text { be distinguished from the cartilage interface sign" [12] }\end{array}$ \\
\hline Aggregates & $\begin{array}{l}\text { "Heterogeneous hyperechoic foci that maintain their high degree of reflectivity even when the gain set- } \\
\text { ting is minimized or the insonation angle is changed and which occasionally may generate posterior } \\
\text { acoustic shadow" [12] }\end{array}$ \\
\hline Tophus & $\begin{array}{l}\text { "A circumscribed, inhomogeneous, hyperechoic and/or hypoechoic aggregation (which may or may not } \\
\text { generate posterior acoustic shadow) which may be surrounded by a small anechoic rim" [12] }\end{array}$ \\
\hline \multicolumn{2}{|l|}{ Non-specific findings } \\
\hline Synovial fluid & $\begin{array}{l}\text { "Abnormal hypoechoic or anechoic (relative to subdermal fat, but sometimes may be isoechoic or hyper- } \\
\text { echoic) intra-articular material that is displaceable and compressible; does not exhibit Doppler signal" } \\
\text { [13] }\end{array}$ \\
\hline Synovial hypertrophy & $\begin{array}{l}\text { "Abnormal hypoechoic (relative to subdermal fat, but sometimes may be isoechoic or hyperechoic) intra- } \\
\text { articular tissue that is nondisplaceable and poorly compressible; may exhibit Doppler signal" [13] }\end{array}$ \\
\hline \multicolumn{2}{|l|}{ Power Doppler signal } \\
\hline $\begin{array}{l}\text { Bone surface changes } \\
\text { (erosions) }\end{array}$ & "An intra- and/or extra-articular discontinuity of the bone surface (visible in 2 perpendicular planes)" [12] \\
\hline
\end{tabular}

Table III. Specific structures for monosodium urate depositions and ultrasonographic findings.

\begin{tabular}{ll}
\hline Anatomical structure & US findings \\
\hline Hyaline cartilage & - Double contour: focal or diffuse enhancement of the superficial articular cartilage layer \\
& - Slightly irregularities of the superficial margin of the hyaline cartilage; \\
Synovial fluid & - Hyperechoic spots $(<1 \mathrm{~mm})$ floating within the joint cavity \\
& - "Snowstorm" appearance \\
Bone & - Intra-articular erosions, profound and destructive \\
Tendon & - Microdeposits: ovoid-shaped hyperechoic densities \\
& - Sntra-tendinous tophi \\
Soft tissue deposits & - Soft tophi: nodular, small, hypoechoic, homogenous structures, soft on palpation \\
& - Hard tophi: hyperechoic band with posterior acoustic shadow, harder in consistency on palpation \\
\hline
\end{tabular}


storm" appearance $[14,15,19]$. Crystal aggregates have to be differentiated from joint debris or proteinaceous materials [19]. Some authors described hyperreflective foci in joint fluids that were related to gas bubbles (probably nitrogen), bubbles formed as a result of joint movement and capsular distension producing fluid agitation [20]. Lowering the gain during examination could help in correct interpretation.

The gold standard for gout diagnosis is the visualization of MSU crystals (in effusion, synovial biopsies, tophi aspirates, etc) [21]. By US the joints with effusions are easily identified and US-guided aspiration can be performed, being of great help for clinicians, especially in undiagnosed cases.

\section{Synovial hypertrophy}

The synovial hypertrophy (fig 1) is a frequent nonspecific finding. The increased vascularisation in the proliferated synovium can be interpreted as inflammatory reaction and may precede erosions and destruction of the adjacent bone [similar with rheumatoid arthritis (RA)], especially if the bone is in contact with a tophus [22]. No specific aspects of the synovitis or vascularisation are seen in order to differentiate the acute gout from other acute arthritis. Only the presence of hyperechoic spots or hyperechoic cloudy areas inside the synovium has high grade of specificity for gout [23]. The presence of the power Doppler signal (especially grade 2 or 3), correlates well with the acutely affected joints. Also, the power Doppler signal disappears with treatment [24]. The pathological vascularisation can be detected also in apparently non-affected joints, demonstrating that power Doppler is significantly more sensitive in identification of the active gouty joints that is the clinical examination [22]. The main utility of US from this point of view seems to be the monitoring of the treatment in gouty patients [25].

\section{Bone erosions}

The bone erosions, step-down lesions in the bone contour detectable in two perpendicular plans, were positive correlated with the number of gout attacks, long disease, and the presence of tophi [26]. Common sites for searching the bone erosions in patients with gout are the medial side of the first metatarsophalangeal (MTP) joint and the metacarpophalangeal (MCP) joints $[12,18,25]$. The erosions in gout detected by US at the MTP-1 are significantly more frequent $(67 \%)$ comparing with other arthritis $(43 \%)$ or healthy subjects $(6 \%)$ [26].

Comparing US with x-ray findings, the erosions suggestive for gout are detected more often by US $[25,26]$ proven to be a moderately specific $(69 \%)$ but with a low sensitivity imaging method (24\%) [27]. The main limitation of the method are the lack of clear criteria for differentiation the gout erosions from erosions of other inflammatory arthritis (RA, spondyloarthritis, etc). The erosions are

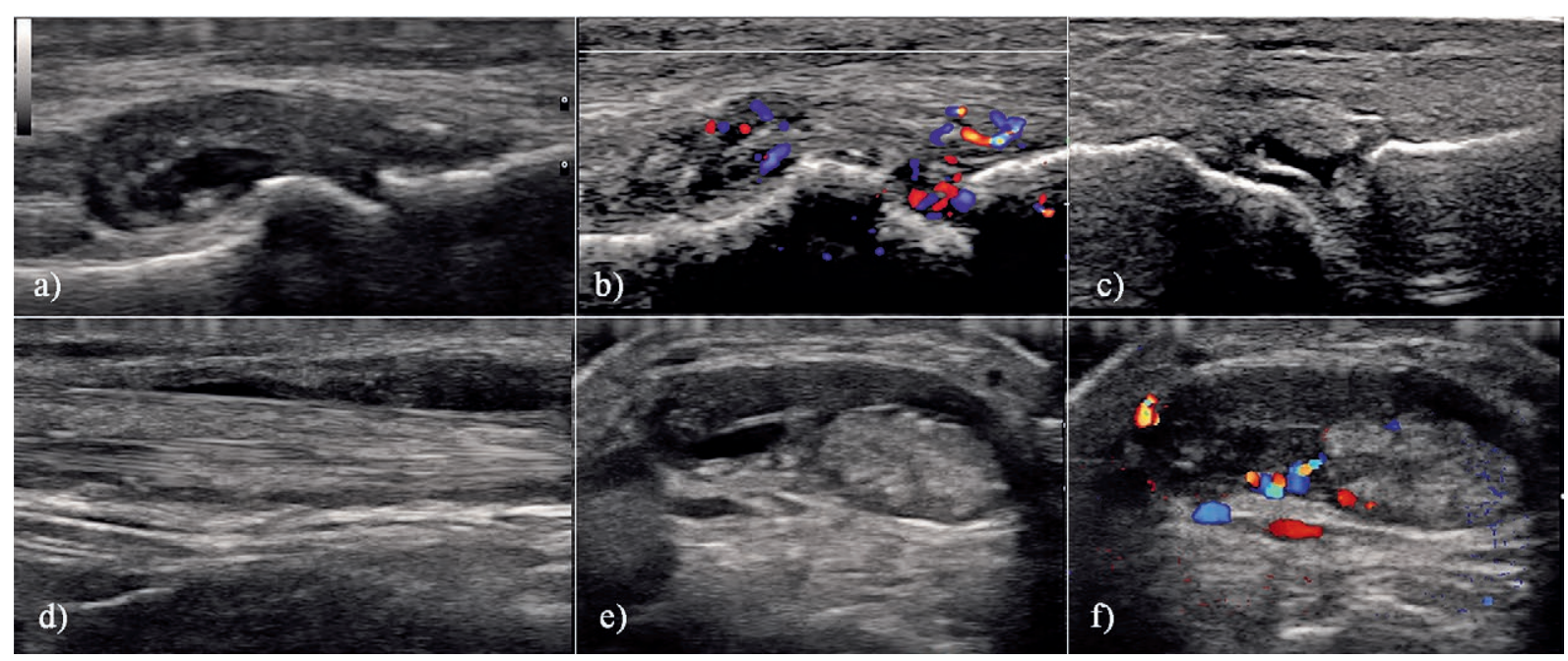

Fig 1. a) Longitudinal scan of the first metatarsophalangeal (MTF) joint- acute gout, gray scale: fluid and proliferated synovium with few hyperechoic spots; b) acute gout- hypervascularised proliferated synovium; c) intercritical gout, second MTF joint - small quantity of transonic fluid and proliferated synovium; note the double contour of the hyaline cartilage; d) longitudinal scan of the tibialis anterior tendon- intratendinous hyperechoic deposits with alteration of the fibrillar structure and tenosynovitis; e) and f) transversal scan of the tibialis anterior tendon, gray scale and color Doppler- hyperechoic deposits, tenosynovitis and hypervascularisation. 
usually more deeper and destructive comparing with RA $[14,27]$ but only the association of erosions with findings suggestive for MSU deposits confer higher specificity.

Extra-articular breaks of the bony cortex due to tophaceous deposits are easily detectable when the deposit is less compact and allows the penetration of the US beam (fig 2, a,b,c).

No standardized US scoring system for erosions in gout exists, but the scoring system created by Wakefield et al [28] for RA (small $<2 \mathrm{~mm}$, moderate $2-4 \mathrm{~mm}$, and large erosions $>4 \mathrm{~mm}$ ) can be applied also for gout.

\section{Articular cartilage}

The US aspect of the normal hyaline cartilage is characterized by a homogeneously anechoic layer delimited by two sharp hyperechoic margins. The superficial margin is thinner than the deeper one and its visualization requires perpendicular ultrasound beam on the cartilage surface (avoiding anisotropy) [19]. MSU have a particular predilection for crystallize on the superficial margin of the cartilage and so the MSU crystals are deposited mainly in the superficial hyaline cartilage. By this mechanism a focal or diffuse enhancement of the superficial margin of the articular cartilage (thickness similar to the subchondral bone), with the reflectivity independent from the angulations of the ultrasound beam - the double contour (DC) sign - is created [11,19,26,29] (fig $2 \mathrm{~d}, \mathrm{e}, \mathrm{f}, \mathrm{g})$. Slightly irregular surface of the hyperechoic band may be seen $[11,19,29]$. This sign is considered to be highly specific for gout $[6,30]$.

The DC sign has $43.6 \%$ sensitivity and $99 \%$ specificity for gout diagnosis [29]. More important, the sign was found only in gouty patients; no other articular disease or healthy subjects had this type of DC, the sign been a useful finding for the differential diagnosis between different arthritides [26]. Moreover, inter-reader reliability in assessing the DC sign was found to be excellent [29,31], making this sign one of the most important US finding in gout.

The DC sign is very well visualized in MTP, knees, MCP and less visualized in joints with thin cartilage (tarsal joints) or with osteoarthritis [6]. It is important to differentiate this sign from the normal cartilage interface (well seen in the presence of the joint effusion due to the posterior enhancement) or from the modifications encountered in osteoarthritis (thin and irregular cartilage) and calcium pyrophosphate deposits (aggregates within the cartilage) $[6,15,19]$.

The presence of the DC sign is not strictly related of the stage of the gout. The sign was found even in the asymptomatic hyperuricemia phase. In a recent study De Miguel et al [32] found DC sign in 27\% (7 patients) of the 26 enrolled patients with asymptomatic hyperuricemia (sensitivity $100 \%$, specificity $88 \%$ ). All the 7 patients had MSU crystals in fluids from joint aspiration. Taking in account EULAR definition [22] these patients should be interpreted as gout despite the lack of symptoms. More studies are necessary to clarify this aspect. In the other phases of gout the DC sign could be consider a marker of disease severity [33], urate levels being positively correlate with this sign in knee and MTF joints (but not with the presence of tophi) [34]. Ottaviani et al [33] found in patients with crystal-proven gout the DC sign with sensitivity of $67 \%$ for MTPs, $57 \%$ for knees, and $21 \%$ for MCPs, and very high specificity ( $>98 \%$ ).

As concerning the responsiveness to treatment, the DC sign disappearance was found to be a good indicator for an efficient treatment [35-37].
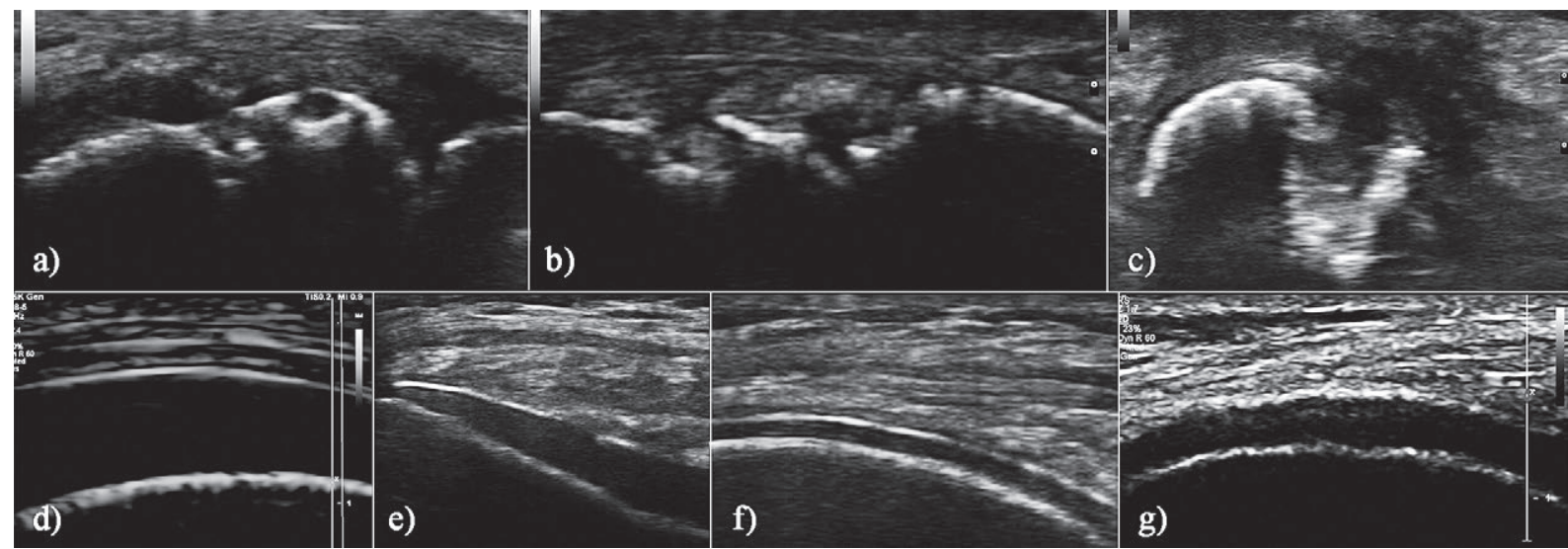

Fig 2. a), b) and c) Small, moderate, and large erosions of the medial part of the first metatarsal head; d) normal aspect of the hyaline cartilage, femoral condyle; e) focal enhancement of the superficial margin of the articular cartilage: the double contour sign; f) diffuse enhancement of the superficial margin of the articular cartilage; g) irregular surface of the hyperechoic band. 
MSU aggregates (hyperechoic spots, hyperechoic cloudy areas, tophi)

At a concentration over $6.8 \mathrm{mg} / \mathrm{dL}$ the serum urate exceeds the limit of solubility and supersaturation of urate in the serum and other extracellular spaces takes place [38]. There are still an-answer questions about the trigger mechanisms of the disease but the deposition of MSU aggregates is consider to be the landmark for gout. These aggregates can be found theoretically in every structure of the organism.

The MSU aggregates reflects ultrasound beams stronger than the surrounding tissues and so it can be easily distinguished and their reflectivity is less influenced by the insonation angle $[6,15,19,38]$. The US aspect of these structures has high variability depending of their size, localizations, distribution, local reactions, or the disease phase [38] (fig 3).

Hyperechoic spot, less than $1 \mathrm{~mm}$, is the most encountered US finding. When hyperechoic spots are found in joint effusion, the "snowstorm" appearance could be demonstrated. Their presence in proliferated synovium is considered to be a specific sign for gout.

Hyperechoic cloudy areas (HCA) or "cottony images" [25] are aggregates less then $1 \mathrm{~cm}$, well or poorly definite, generally homogeneous without posterior acoustic shadow [39] and are considered to be elementary lesions in gout [35]. HCA were found to be significantly influenced by treatment [35].

The tophi, extracellular deposits of MSU surrounded by foreign-body giant cells and mononuclear cells forming a granuloma-like structure [5], have US aspect depending on their age and size. Tophi may occur at any site but their frequent locations are the toes, especially
MTP I (dorsal more frequent that volar), wrist, fingers, knee, and ankle $[17,18]$. Three types of tophi were described according to the degree of compaction of the deposits: soft, hard, and mixed [14,15,19,40].

At the beginning the tophi are nodular, small, hypoechoic, with homogenous structure, and soft on palpation - soft tophi. A hyperechoic rim could be found [7]. As they grow they become more echogenic, with hyperechoic edges and well differentiated from the surrounding tissues. They can dislocate the normal structures of the area or even compress them (tendons, vessels, nerves, bone). For example there are cases with carpal tunnel syndrome caused by tophaceous gout [41]. In this stage a clear differentiation from rheumatoid nodules is difficult [21]. Sonoelastography was recently used to differentiate rheumatoid nodules from tophi and the result are promising [42].

After years the tophi might develop into pseudotumoral forms and become dishomogenous, harder in consistency on palpation - hard or mixed tophi, even with calcification foci, with posterior acoustic shadow. A hypoechoic halo can appear if there is an inflammatory reaction around the tophus. This rim is formed by macrophages, lymphocytes and large foreign body giant cells [43] and if this kind of tophus is in contact with the bone, the presence of the inflammatory cells can explain why the tophus erodes the bone surface [11]. In patients with gouty flare a surrounding edematous and hypervascularised soft tissues is observed [21]. Moreover, a persistent low-grade of inflammation in asymptomatic chronic tophaceous gouty arthritis was found and this may explain the silent development of erosions [44].

The prevalence of tophi in gout becomes higher during the disease evolution. Filippucci et al [18] found nearly half of the gouty patients having tophi in at least

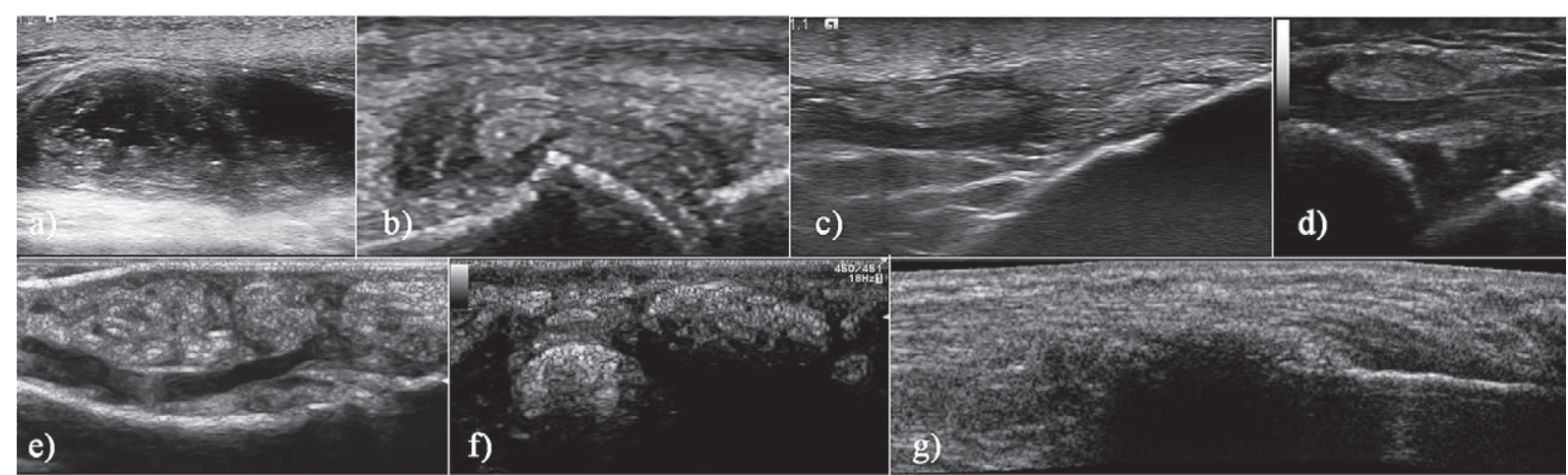

Fig 3. Monosodium urate deposits: a) hyperechoic spots, less than $1 \mathrm{~mm}$ in the proliferated synovium of the suprapatelar bursa of the knee; b) hyperechoic spots and hyperechoic cloudy areas in the first metatarsophalangeal joint, dorsal longitudinal scan; c) hyperechoic cloudy areas in the patellar tendon, longitudinal scan; d) hyperechoic cloudy area in the synovial sheath of the tibialis anterior tendon, longitudinal scan; note the fluid in the ankle joint; e) soft tophi in the middle and distal phalanx, volar scan; f) mixed tophi near the elbow; g) hard tophus in the Achilles tendon. 
one of the examined joints. Ottaviani et al [34] detected articular urate deposits in $60 \%$ of gouty patients (the prevalence of tophi for knees and MTPs being 26.7\%), more common in patients with higher serum uric acid. The tophi were found as well in patients with asymptomatic hyperuricaemia not only in synovium but also in tendons and soft tissue $[45,46]$.

As concerning the measurement of the tophi PerezRuiz et al [47] compared the utility of MRI and US in evaluating intraarticular and articular tophi. US detected at least one tophus in all joints but MRI found nodules considered to be tophi. The aspiration of nodules suspected as tophi confirmed MSU crystals in $83 \%$. The study found good correlation but only fair agreement between US and MRI. More important is the higher intraobserver intraclass correlation for diameters and volume, suggesting that US is a reliable method for measure the tophi. This aspect is important in monitoring the gout evolution and response to treatment. In patients with adequate control of serum uric acid levels a decreased in diameter and volume of the tophi was observed [47].

Intratendinous tophi can be frequently found inside the patellar ligament, Achilles, triceps, quadricipital, anterior tibialis tendons, and plantar fascia. They are hypo/ hyperechoic and inhomogeneous, with poorly defined margins, producing focal alteration of the fibrillar structure of the tendon and generating posterior shadow. Hyperechoic linear bands deposits of MSU within the tendon substance were also described [6,15,17-19,48,49]. These lesions are areas of minimal resistance and spontaneous tears can occur [50].

Among the periarticular bursae, the pre-patellar and olecranon bursae are the most commonly involved sites $[46,49]$. Fernandes et al [51] found that hyperechogenicity $(91.7 \%)$, poorly defined contours $(88.6 \%)$, multiple grouped nodules $(85.6 \%)$, and heterogeneity $(68.6 \%)$ are the most characteristics findings of the tophi from olecranon bursa. Peritendinous bursae (infrapatellar, preachillian) were found to be inflamed in cases with concommitant tendon pathology [16].

Subcutaneous tophi should be differentiated from other types of nodules, especially rheumatoid nodules. In $80 \%$ of the gouty nodules the masses are heterogeneous and hyperechoic $[27,52]$.

The complex deposits of MSU in digits (tendon, subcutaneous tissue, etc) could create the aspect of gouty dactylitis, a rare finding, seen in chronic polyarticular disease [53-55].

Enthesopathy, although uncharacteristic for gout, was found around patella, quadriceps tendon, and patellar ligament insertions and around calcaneus at Achilles tendon and plantar fascia insertions [56].

\section{Investigation questions}

One of the problems in US investigation of gouty patients is which sites to be investigated (only symptomatic joints or structures or more extensive examination) and what findings to be search (MSU deposits, effusion, synovial hypertrophy, vascularisation) in order to have good sensibility and specificity of the method.

In a recent published paper by Naredo et al [17] the authors found that US examination of 12 anatomical sites (bilateral radio-carpal joint, first metatarsal head cartilage, talar cartilage, second metacarpal head, knee femoral condyle cartilage, patellar and triceps tendons) for DC sign and hyperechoic aggregates gives the best results for sensitivity and specificity $(84.6 \%$ and $83.3 \%$, respectively). This approach had high positive and good negative predictive values ( $92 \%$ and $71 \%$, respectively) for diagnosing gout.

Peiteado et al [57] studied the presence of six types of lesions in gouty patients: hyperechoic spots in the synovial fluid, HCA, bright stippled aggregates, DC sign, erosions, and the Doppler signal in 17 joints and 8 tendons. They found that the knees and MTP joints are the most frequent affected sites (in $93 \%$ of patients) and a six-minute US examination of four joints (knees and the 1st MTPs) allowed the detection of HCA or DC sign in $97 \%$ of cases.

Clinical diagnosis of gout is sometimes difficult, even if the manifestations are in MTF-1 (classical podagra). Kienhorst et al [58] recently demonstrated that in patients with MTP-1 arthritis the gout was the correct diagnosed only in $77 \%$ of cases but the general practitioner supposed gout in $98 \%$ of cases. In these cases US examination of the joint could simplify the diagnosis process. This fact was demonstrated by Lamers-Karnebeek et al [59] in patients with monoarthrithis in which $48 \%$ of patients had MSU crystals-proven gout. In this cases sensitivity of DC sign and any US abnormality (DC sign, tophi, or snowstorm appearance of the joint effusion) was 77 and $96 \%$, respectively. The authors considered that the DC sign is an important informative finding for clinician, with positive predictive value of $74 \%$ and negative predictive value of $78 \%$.

The new US techniques probably will increase the US capacity to detect the crystals aggregates. MicroPure, a new US image processing function designed to improve the visualization of microcalcifications, was used by Yin et al [60] in patients with gout. Significantly more microcalcifications were seen with MicroPure compared to gray scale US and the level of agreement between investigators was consistently improved. The method seems to be useful especially for the cases where the presence of gray scale US artifacts or unspecific findings make 
difficult the interpretation. The use of different imaging methods (dual energy CT, MRI, positron emission tomography) may improve the description of lesions [61]. The main question is if there is a really need in clinical practice for such a complex approach.

The need for good education and training for identification and interpretation of the US findings encountered in gout was underline by Filippucci et al [62].The authors highlight the necessity for dedicated training-programme to avoid the false positive and false negative results. After 7 days of training the rheumatologists with limited US experience gain satisfactory skills to identify MSU aggregates in different tissues.

Due to the lower diagnostic utility of the clinical gouty features (exception tophi and response to colchicine) [63], there is an incremental need for imaging techniques to confirm the clinicians' suppositions. The place of the US in patients suspected of gout must be clearly defined [59] due to the high numbers of patients presented with monoarthritis. The research agenda concerning the use of US in gouty patients is still busy despite of the recent achievements in this pathology.

In conclusion in the assessment of the gouty patients US is an important and valuable tool. For a complete examination and a correct interpretation of the imagines the examiner need to have solid US knowledge about normal and pathological aspects of the musculoskeletal structures. Knowing the specific US aspects of MSU depositions will permit a rapid diagnosis. Moreover, when combining the clinical examination with US scan in patients with suspicion of gout a proper and rapid decisions for the patient management can be taken.

\section{Conflict of interest: none}

\section{References}

1. Robinson PC, Horsburgh S. Gout: joints and beyond, epidemiology, clinical features, treatment and co-morbidities. Maturitas 2014; 78: 245-251.

2. Sivera F, Andrés M, Carmona L, et al. Multinational evidence-based recommendations for the diagnosis and management of gout. Ann Rheum Dis 2014; 73: 328-335.

3. Girish G, Glazebrook K, Jacobson J. Advanced Imaging in Gout. AJR Am J Roentgenol 2013;201:515-525.

4. Dalbeth N, Stamp L. Hyperuricaemia and gout: time for a new staging system? Ann Rheum Dis 2014; 73: 1598-1600.

5. Kelly WN, Schumacher HR. Gout. In Kelly-Harris-RuddySledge Textbook of Rheumatology 4th ed. WB Saunders Company, 1993: 1291-1330.

6. Ottaviani S, Bardin T, Richette P. Usefulness of ultrasonography for gout. Joint Bone Spine 2012; 79: 441-445.
7. Gerster JC, Landry M, Dufresne L, Meuwly JY. Imaging of tophaceous gout: computed tomography provides specific images compared with magnetic resonance imaging and ultrasonography. Ann Rheum Dis 2002; 61: 52-54.

8. Gerster JC, Landry M, Duvoisin B, Rappoport G. Computed tomography of the knee joint as an indicator of intraarticular tophi in gout. Arthritis Rheum 1996; 39: 1406-1409.

9. Mallinson PI, Reagan AC, Coupal T, Munk PL, Ouellette $\mathrm{H}$, Nicolaou $\mathrm{S}$. The distribution of urate deposition within the extremities in gout: a review of 148 dual-energy CT cases. Skeletal Radiol 2014; 43: 277-281.

10. Popovich I, Dalbeth N, Doyle A, Reeves Q, McQueen FM. Exploring cartilage damage in gout using 3-T MRI: distribution and associations with joint inflammation and tophus deposition. Skeletal Radiol 2014; 43: 917-924.

11. Thiele RG, Schlesinger N. Diagnosis of gout by ultrasound. Rheumatology 2007; 46: 1116-1121.

12. Gutierrez M, Smith W, Thiele R, et al. Defining elementary ultrasound lesions in gout. preliminary results of delphi consensus and web-exercise reliability. Ann Rheum Dis 2014; 73(Suppl2).

13. Wakefield RJ, Balint PV, Szkudlarek M, et al. Musculoskeletal ultrasound including definitions for ultrasonographic pathology. J Rheumatol 2005; 32: 2485-2487.

14. Thiele RG. Ultrasound in the diagnosis of crystals deposition diseases. In: Wakefield RJ, D’Agostino MA (eds). Essential applications of musculoskeletal ultrasound in rheumatology. Saunders Elsevier, 2010: 331-343.

15. Delle Sedie A, Riente L, Iagnocco A, et al. Ultrasound imaging for the rheumatologist X. Ultrasound imaging in crystal-related arthropathies. Clin Exp Rheumatol 2007; 25: 513-517.

16. Fodor D, Albu A, Gherman C. Crystal Associated Synovitis - Ultrasonographic Feature and Clinical Correlation. OTR 2008; 2; 99-110.

17. Naredo E, Uson J, Jiménez-Palop M, et al. E. Ultrasounddetected musculoskeletal urate crystal deposition: which joints and what findings should be assessed for diagnosing Gout? Ann Rheum Dis 2014; 73: 1522-1528.

18. Filippucci E, Meenagh G, Delle Sedie A, et al. Ultrasound imaging for the rheumatologist XXXVI. Sonographic assessment of the foot in gout patients.Clin Exp Rheumatol 2011;29: 901-905.

19. Grassi W, Meenagh G, Pascual E, et al. 'Crystal clear'-sonographic assessment of gout and calcium pyrophosphate deposition disease. Semin Arthritis Rheum 2006; 36: 197 202.

20. Wakefield R, O`Connor P. All that glimmers is not gold. Semin Arthritis Rheum 2007; 37: 133-134.

21. Schueller-Weidekamm C, Schueller G, Aringer M, Weber M, Kainberger F. Impact of sonography in gouty arthritis: comparison with conventional radiography, clinical examination, and laboratory findings. Eur J Radiol 2007; 62: 437443.

22. Zhang W, Doherty M, Pascual E, et al. EULAR evidence based recommendations for gout. Part I: Diagnosis. Report of a task force of the standing committee for international 
clinical studies including therapeutics (ESCISIT). Ann Rheum Dis 2006;65; 1301-1311.

23. Perez-Ruiz F, Urresola A, Miguel E, Schlesinger N. Gout. Imaging of gout: findings and utility. Arthritis Res Ther 2009; 11: 232.

24. Filippucci E, Capetti A, Grassi W. Sonographic monitoring of gout. Reumatismo 2003; 55: 184-186.

25. Villaverde V, Rosario MP, Loza E, Pérez F. Systematic review of the value of ultrasound and magnetic resonance musculoskeletal imaging in the evaluation of response to treatment of gout. Reumatol Clin 2014; 10: 160-163.

26. Wright SA, Filippucci E, McVeigh C, et al. High resolution ultrasonography of the 1st metatarsal phalangeal join in gout: a controlled study. Ann Rheum Dis 2007; 66: 859864.

27. Rettenbacher T, Ennemoser S, Weirich H, et al. Diagnostic imaging of gout. Comparison of high-resolution US versus conventional X-ray. Eur Radiol 2008; 18: 621-630.

28. Wakefield RJ, Gibbon WW, Conaghan PG, et al. The value of sonography in the detection of bone erosions in patients with rheumatoid arthritis: a comparison with conventional radiography. Arthritis Rheum 2000; 43: 2762-2770.

29. Filippucci E, Riveros MG, Georgescu D, Salaffi F, Grassi W. Hyaline cartilage involvement in patients with gout and calcium pyrophosphate deposition disease: An ultrasound study. Osteoarthritis Cartilage 2009; 17: 178-181.

30. Chowalloor PV, Keen HI. A systematic review of ultrasonography in gout and asymptomatic hyperuricaemia. Ann Rheum Dis 2013; 72: 638-645.

31. Howard RG, Pillinger M, Gyftopoulos, Thiele R., Swearingen C, Samuel S. Reproducibility of musculoskeletal ultrasound for determining monosodium urate deposition:concordance between readers. Arthritis Care Research 2011; 63; 1456-1462.

32. De Miguel E, Puig JG, Castillo C, Peiteado D, Torres RJ, Martín-Mola E. Diagnosis of gout in patients with asymptomatic hyperuricaemia: a pilot ultrasound study. Ann Rheum Dis 2012; 71: 157-158.

33. Ottaviani S, Richette P, Allard A, Ora J, Bardin T. Ultrasonography in gout: a case-control study. Clin Exp Rheumatol 2012; 30: 499-504.

34. Ottaviani S, Allard A, Bardin T, Richette P. An exploratory ultrasound study of early gout. Clin Exp Rheumatol 2011; 29: 816-821.

35. Peiteado D, Villalba, D, de Miguel E, Ordonez MC, MartinMola E. Logitudinal study of ultrasonography sensibility to change in patients with gout after one year of treatment. Ann Rheum Dis 2012; 69(Suppl 2): 713.

36. Perez-Ruiz F, Martin I, Canteli B. Ultrasonographic measurement of tophi as an outcome measure for chronic gout. J Rheumatol 2007; 34: 1888-1893.

37. Thiele RG, Schlesinger N. Ultrasonography shows disappearance of monosodium urate crystal deposition on hyaline cartilage after sustained normouricemia is achieved. Rheumatol Int 2010; 30: 495-503.

38. Olivieri F, Scanu A, Punzi L. Metabolism of crystals within the joint. Reumatismo 2012; 63: 221-229.
39. Filippucci E, Di Geso L, Grassi W. Tips and tricks to recognize microcrystalline arthritis. Rheumatology (Oxford) 2012; 51(Suppl 7): vii18-21.

40. Filippucci E, Di Geso L, Girolimetti R, Grassi W. Ultrasound in crystal-related arthritis. Clin Exp Rheumatol 2014; 32(Suppl 80): S42-47.

41. Chen CK, Chung CB, Yeh L, et al. Carpal tunnel syndrome caused by tophaceous gout: CT and MR imaging features in 20 patients. ARJ Am J Roentgenol 2000; 175: 655-659.

42. Sconfienza LM, Silvestri E, Bartolini B, Garlaschi G, Cimmino MA. Sonoelastography may help in the differential diagnosis between rheumatoid nodules and tophi. Clin Exp Rheumatol 2010; 28: 144-145.

43. Rosenberg AE. Bones, Joints, and Soft Tissue Tumors. In: Kumar V, et al (Eds). Robbins and Cotran Pathologic Basis of Disease. Elsevier 2005: 1311-1314.

44. Schlesinger N, Thiele RG. The pathogenesis of bone erosions in gouty arthritis. Ann Rheum Dis 2010; 69: 1907-1912.

45. Pineda C, Amezcua-Guerra LM, Solano C, et al. Joint and tendon subclinical involvement suggestive of gouty arthritis in asymptomatic hyperuricemia: an ultrasound controlled study. Arthritis Res Ther 2011; 13: R4.

46. Puig JG, de Miguel E, Castillo MC, et al. Asymptomatic hyperuricemia: impact of ultrasonography. Nucleosides Nucleotides Nucleic Acids 2008; 27: 592-595.

47. Perez-Ruiz F, Martin I, Canteli B. Ultrasonographic measurement of tophi as an outcome measure for chronic gout. J Rheumatol 2007; 34: 1888-1893.

48. Abate M, Schiavone C, Salini V, Andia I.Occurence of Tendon Pathologies in Metabolic Disorders. Rheumatology 2013; 52: 599-608.

49. Rodas G, Pedret C, Catala J, Soler R, Orozco L,Cusi M. Intratendinous Gouty Tophus Mimics Patellar Tendonitis in an Athlete. J cLin Ultrasound 2013; 41: 178-182.

50. Mahoney PG, Lames PD, Howell CJ, et al. Spontaneous rupture of the Achilles tendon in a patient with gout. Ann Rheum Dis 1981; 40: 416-418.

51. Fernandes EA, Lopes MG, Mitraud SA, Ferrari AJ, Fernandes AR. Ultrasound characteristics of gouty tophi in the olecranon bursa and evaluation of their reproducibility. Eur J Radiol 2012; 81: 317-323.

52. Nalbant S, Corominas H, Hsu B, et al. Ultrasonography for Assessment of Subcutaneous Nodules. J Rheumatol 2003; 30: 1191-1195.

53. Fodor D, Lungu A. Ultrasonography of the non-traumatic lesions of the fingers. Pictorial essay. Med Ultrason 2013; 15: 147-153.

54. Healy PJ, Helliwell PS. Dactylitis: pathogenesis and clinical considerations. Curr Rheumatol Rep 2006; 8: 338-341.

55. Olivieri I, Scarano E, Padula A. Dactylitis, a term for different digit diseases. Scand J Rheumatol 2006; 35: 333-340.

56. Gerster JC, Landry M, Rappoport G, Rivier G, Duvoisin B, Schnyder P. Enthesopathy and tendinopathy in gout: computed tomographic assessment. Ann Rheum Dis 1996; 55: 921-923.

57. Peiteado D, De Miguel E, Villalba A, Ordóñez MC, Castillo C, Martín-Mola E. Value of a short four-joint ultrasound 
test for gout diagnosis: a pilot study. Clin Exp Rheumatol 2012; 30: 830-837.

58. Kienhorst LB, Janssens HJ, Fransen J, van de Lisdonk EH, Janssen M. Arthritis of the first metatarsophalangeal joint is not always gout: a prospective cohort study in primary care patients. Joint Bone Spine 2014; 81: 342-346.

59. Lamers-Karnebeek FB, Van Riel PL, Jansen TL. Additive value for ultrasonographic signal in a screening algorithm for patients presenting with acute mono-/oligoarthritis in whom gout is suspected.Clin Rheumatol 2014; 33: 555559.

60. Yin L, Zhu J, Xue Q, et al. MicroPure imaging for the evaluation of microcalcifications in gouty arthritis involving the first metatarsophalangeal joint: a preliminary study. PLoS One 2014; 9: e95743.

61. Su H, Li X, Zhao N, Zou H, Jiang L, Zhou Y. Comparing the usefulness of four imaging techniques in the same gouty arthritis patient. Int J Rheum Dis 2012; 15: e128-130.

62. Gutiérrez M, Di Geso L, Rovisco J, et al. Ultrasound learning curve in gout: a disease-oriented training program. Arthritis Care Res (Hoboken) 2013; 65: 1265-1274.

63. Sivera F, Andrès M, Falzon L, van der Heijde DM, Carmona L. Diagnostic value of clinical, laboratory, and imaging findings in patients with a clinical suspicion of gout: a systematic literature review. J Rheumatol Suppl 2014; 92: 3-8. 